\title{
Contribuciones al conocimiento de la flora de Asturias, II
}

\author{
C. AEDO, J. M. ARGÜELLES, J. M. GONZÁLEZ DEL VALLE \& M. LAÍNZ
}

\begin{abstract}
Aedo, C., J. M. Argúelles, J. M. González del Valle \& M. Laínz (1990). Contributions to the knowledge of the flora of Asturias, II. Collect. Bot. (Barcelona) 18: 99-116.

We are progressing in our prolonged floristic work - v. ARGÚELLES \& al. (1984), AEDO \& al. (1989), LAínZ (1982), etc.-, regarding in one way or another the Cantabrian zone in the most traditional and ample sense, as the political or administrative views would seem secondary in the case. Of course we are not going to evaluate here our present contributions, so many and diversified in fact. What matters most is to go on working systematically and to publish everything as it has been done so far. Hieracium saliencianum de Retz, sp. nov.
\end{abstract}

Keywords: Vascular plants, Taxonomy, Asteraceae, Hieracium, Chorology, North West Spain.

\section{Resumen}

Aedo, C., J. M. Argüelles, J. M. González del Valle \& M. Laínz (1990). Contribuciones al conocimiento de la flora de Asturias, II. Collect. Bot. (Barcelona) 18: 99-116.

Llevamos adelante nuestro prolongado trabajo florístico - v. ARGUELLES \& al. (1984), AEDo \& al. (1989), LAinz (1982), etc.-, referente de uno u otro modo a la zona cantábrica en el sentido amplio y tradicional, ya que las consideraciones político-administrativas podrían parecer muy secundarias en el asunto. No haremos aquí balance de nuestras presentes contribuciones, harto nutridas, bien que de naturaleza diversa, claro está. Lo que importa es continuarlas de manera sistemática, publicándolas con todos los oportunos detalles, como hemos venido siempre haciéndolo. Hieracium saliencianum de Retz, sp. nov.

Palabras clave: Plantas vasculares, Taxonomía, Asteraceae, Hieracium, Corología, Noroeste de España.

Selaginella kraussiana (G. Kunze) A. Braun

Pancar y La Pereda (Llanes), 30TUP50, sitio umbrío - bajo una higuera, entre ortigas-y en otro asimismo inculto, más lejos aún de poblado, a 20 y 60 m. F. J. López de Saro leg., 
28-VII-1988 y VII-1989 de modo respectivo. Especie africana que no se había señalado hasta el momento en Asturias.

\section{Equisetum variegatum Schleicher}

Aguazales de la famosa Vega de Liordes, provincia de León, 30TUN57, a $1985 \mathrm{~m}$. Reconocida por nosotros el 10-VII-1988; y colectada, en buenas condiciones, el 2-IX-1988, en la grata compañía de P. Montserrat, L. Villar, D. Gómez y Asunción García González. Relevante novedad para los Picos de Europa, bien que señalada no hace mucho (DíAz \& PUENTE, 1985: 269, 289), de forma concreta, en la zona leonesa de Somiedo: 29TQH26. Las citas cantábricas previas eran las de 1961 y 1970, entrambas de Asturias, 30TTN66 y 29TQH37 de modo respectivo (cf. LAİNZ, 1982: 19).

\section{E. silvaticum L. [«sylvaticum»]}

Tras no poco esfuerzo, por fin dimos -Argüelles y Laínz, 15-IX-1985, ad 1400 m- con una colonia sumamente nutrida, la misma, seguro, que vio Lagasca en 1803 (cf. LAÍNZ, 1982: 20). Se ha depositado material en diversas instituciones. Queda también clarísimo que la otra cita leonesa tenía base firme (cf. LlamAS \& al., 1985: 283). Por el contrario, ha fracasado hasta hoy nuestro empeño de ver en Asturias - buscándola muy a fondo en la divisoria- la reliquia en cuestión. Seguimos dando por cierto (cf. LAÍNZ, 1970: 8) que los materiales de «Mieres» no son españoles. Y recordemos a este propósito que también habría dado Lázaro en Mieres con lo que se llama en «Flora iberica» Thelypteris palustris Schott (cf. CoLMEIRO, 1889: 453), la que hasta hoy ha seguido eludiendo a todos en Asturias: otra superchería de tantas como hubimos ya de señalar. En cuanto a las indicaciones del APIB para la especie que nos ocupa (cf. SALVO \& al., 1984: 110), olvídense tanto la montañesa -idependiente aún de lo demás que imprimió Colmeiro?- como la navarra, que se basaba en cita (BÁSCONES \& al., 1982: 23) que ha resultado ser errónea (cf. CATALÁN \& AIZPURU, 1989:101).

Y aclaremos hoy (cf. LAÍNZ, 1979: 32), continuando un viejo post scriptum (cf. LAÍNZ, 1968: 38), que la cita regional de «Cystopteris montana» tuvo por base una mala interpretación de la nota manuscrita de Bory: concreta, cierto, «hauteurs de Mezana» (Boal), pero tras epíteto, «montanum», que se refiere al nombre genérico «Nephrodium»... Siguiéndose de ahí que tal cita se ha de llevar a lo que «Flora iberica» llama Lastrea limbosperma (All.) J. Holub \& Pouzar, con lo que su verosimilitud se hace incuestionable.

\section{Polystichum $\times$ illyricum (Borbás) Hahne}

Bajo el Forao -la «Peñafurada» lagascana- de Viadangos (Villamanín, León), 30TTN75, Delgado, Laínz \& Torío leg., 13-VII-1986. Allí, donde abundan los progenitores $-P$. aculeatum (L. ) Roth y $P$. lonchitis (L.) Roth-, pudimos convencernos de que son erróneas las citas más o menos comarcales de $P$. setiferum (Forsskål) Woynar hechas o recogidas por DíAZ \& PUENTE (1985: 278-279, 296), quienes confiaban, a no dudar, en diagnosis un tanto simplistas que piden finura en la interpretación. Ese mapa debe revisarse, así como determinaciones posteriores que siguen su línea (cf. HERRERo \& al., 1988: 369; PUENTE \& al., 1988: 373).

La muestra del híbrido que decimos parece muy clara. Cierto que, sin tener a la vista esporangios maduros, quedan siempre, frente a él, de no recurrirse a otros métodos, inseguridades. Luego, con H. Nava, el 3-IX-1987, se colectó por encima de la Vega de Meicín (Lena, macizo de Ubiña), 30TTN56, algo que podrá llevarse al mismo, tampoco fértil y morfológicamente más dudoso. 


\section{Polygonum amphibium L.}

Junto a la playa de Penarronda (Castropol-Tapia de Casariego), 29TPJ62, Laínz, 5-VII-1968, y en las cercanías de Villadún (Barres, Castropol), sitio muy próximo, Aedo, 4-V-1989. A un lado la vaguedad que se puede ver en Colmeiro, conocemos la cita picoeuropeana de GUINEA (1953: 373), referente al célebre lago de la Ercina, 30TUN39, y bien confirmada por FERNÁNDEZ BERNALDO DE QUIRÓs \& GARCÍA FERNÁNDEZ (1987: 242), más la de Silvota (Llanera), 30TTP72, que figura en la pág. 246 de los mismos autores, y otra de Vega de Anzo (Grado), 29TQJ40, ésta de LASTRA \& MAYOR (1980: 310). En todo caso, raro en la Cornisa Cantábrica; y desaparecido ya de su localidad santanderina (cf. AEDo \& al., 1984: 127), por desecación de aquellos terrenos. MERINo (1906: 545-546) lo citaba de Galdo (Vivero, Lugo).

\section{Rumex pseudalpinus Höfft}

Su recolección primera en Asturias (cf. NAVA \& VERA DE LA PUENTE, 1987: 536) tiene todos los visos de haberse realizado en localidad no primaria, interesantísima sin duda. Cf. LAÍNZ, 1987b: 603; LÓPEZ GONZÁLEZ, 1988: 368-369. Se hace una ulterior cita montañesa, quinta cantábrica cierta, en AEDo \& al. (en prensa). Leonesas, del valle de Valdeón, Aedo hace ahora las cuatro indicaciones - observaciones- que siguen: herbazales de siega en el Gildar, 30TUN47, como a 1600 m, 18-VIII-1989; Caldevilla, 30TUN47, y Cordiñanes, 30TUN48, sendos ejemplares gigantescos, en la orilla del Cares, a 980 y 840 m respectivamente, 4-IX-1989; más laderas de la Peña de la Silla, 30TUN47, junto a un arroyo, a $1460 \mathrm{~m}$, 12-IX-1989.

\section{R. aquitanicus Rech. fil., syn. $R$. cantabricus Rech. fil.}

Por concretar alguna localidad asturiana (cf. LAİNZ, 1979: 34), señalemos hoy la recoleción hecha sobre Bulnes (Cabrales), 28-VII-1968, herbazal de siega junto a la Terenosa, 30TUN48, como a $1300 \mathrm{~m}$; más otra de Aedo, 27-VI-1986, hecha pr. Páramo (Teverga), 29TQH47, donde también se comporta la planta como pratense, a $1200 \mathrm{~m}$. Luego, el 21-VI-1989, Aedo lo ha visto sobre Río-Aller (San Román de Casomera, concejo de Aller), 30TUN07, y tan solo con hojas en la misma fecha y cuadrícula, más arriba $-1500 \mathrm{~m}-$, en el puerto de Vegarada. Finalmente -ya en León-, el 29-VI-1989 lo ha colectado en Villablino, 29TQH17, dentro del pueblo. En cuanto a nomenclatura, cf. LÓPEZ GONZÁLEZ, 1987: 585.

\section{Carpobrotus acinaciformis (L.) Bolus}

Parece ser la especie del género que asimismo - como en la vecina Cantabria (cf. AEDo \& al., en prensa) - se cultiva y, en dunas o acantilados, echa pie firme aquí en Asturias: v. gr., en los arenales de San Esteban de Pravia, 29TQJ32, Aedo, 12-II-1989, y al final del paseo marítimo de Gijón, 30TTP82, Laínz, 8-III-1989. Cierto que nuestra planta no es glauca, en contra de lo que afirman algunas descripciones, y que la total de $C$. edulis (L. ) N. E. Br. recién detallada por PRESTON \& SELL (1989: 237-241) aclara poco las cosas. En última hipótesis, acinaciformis tendría frente a edulis prioridad en el rango específico; aunque todo el mundo parece darlos por especies diversas, muy variables por lo visto. Las hojas de nuestra planta son «acinaciformes»-o sea, muy comprimidas lateralmente-y no triquetras a secas; las flores, purpúreas. En DíAZ (1977: 131), lo que se da por asturiano es edulis, aunque sin referirse a su caracterización, salvo de modo indirectísimo. 
Stellaria nemorum L. subsp. montana (Pierrat) Berher

Especie que recientemente señalábamos, de manera poco visible (cf. LAÍNZ, 1987b: 603), como novedad asturiana. Sobre nombre correcto de la subespecie, cf. Lejeunia 120: 170-171 (1987).

\section{Dianthus langeanus Willk.}

Hemos de referirnos al mismo para recortar una vez más ampliaciones erróneas de su área -cf. ARGÜELles \& al., 1984: 6-, diciendo ahora que no son correctas las citas del Pico Huevo de Faro, etc. (LÓPEz PACHECO, 1988: 85), y de Braña Caballo (VERA DE LA PUENTE, 1985: 76, cuadro frente a dicha página, sin paginar). Ahí, como en el Cellón, las plantitas que nosotros hemos visto una y otra vez, aunque silicícolas de ordinario, tienen brácteas caliculares muy netamente mucronadas y hojas bien diversas de las de la especie occidental; por lo que deberán llevarse a D. pungens L. subsp. brachyanthus (Boiss.) Bernal, Fernández Casas, G. López, Laínz \& Muñoz Garmendia, salvo que se arbitre solución mejor.

PÉREZ MoRAles (1988: 66) reitera, por cierto, cita como la de su «D. hispanicus Asso var. borealis Willk. ", de Santa Lucía (cf. ARGÜELLES \& al., 1984: 6); bien que añadiendo para esa misma localidad y otras la de su «D. subacaulis Vill. subsp. brachyanthus (Boiss. ) P. Fourn. », más de acuerdo con todas nuestras observaciones comarcales y locales. ¿Aparecerán ahí realmente cálices que alcancen los milímetros de longitud exigibles a D. pungens subsp. hispanicus (Asso) O. de Bolòs \& Vigo, con dientes puntiagudos? Más inverosímil aún es que se lo haya visto en Quintanilla de Flórez (Quintana y Congosto, León), 29TQG38, donde vuelve a señalarse la «var. borealis» (GARCÍA GoNZÁLEZ, 1988: 127), como si tal cosa.

Petrocoptis glaucifolia (Lag.) Boiss. subsp. wiedmannii (Merxmüller \& Grau) D. Fernández González, T. E. Díaz, M. A. Fombella \& R. M. Valencia, Lagascalia 15 (extra): 219 (1988)...

Combinación propuesta en el seno de un trabajo de reducidos horizontes palinológicos; aunque, según se declara, estribando en «estudio llevado a cabo sobre numerosas poblaciones cantábricas, que en principio se podrían asignar a dicho taxón». Como carácter esencial frente a glaucifolia, se invoca la glabricie de la nueva subsp. wiedmannii - o más bien, status nuevo-, admitiendo la existencia de transiciones. El «apéndice» de la página última -suponemos que se mencionan ahí todos los pliegos cuyo polen ha sido estudiado-incluye tan solo uno para cada uno de los bendecidos táxones. Por otra parte, MONTSERRAT (1988: 362) toma, de acuerdo con Fernández Casas, decisiones muy diversas, que no discutiremos. Lo cierto es, para nosotros (cf. LAÍNZ, 1974a: 190-193), que la pilosidad foliar se relaciona con la sequedad ambiental a que Montserrat alude: frente al original provisorio de «Flora iberica» nos hemos preguntado en qué se diferencian esencialmente las subespecies glaucifolia (Lag.) P. Monts. $\&$ Fernández Casas y pyrenaica, supeditadas por los autores del mismo a la prístina $P$. pyrenaica (J. P. Bergeret) A. Braun...

\section{Agrostemma githago L.}

Pr. Corao (Cangas de Onís), 30TUP30, campo de avena, leg. Aedo, 25-V-1986. Su más próxima localidad conocida sería la casina de MAYOR \& al. (1982: 12), 30TUN08. FERNÁNDEZ PRIETO \& al. (1982: 27) la citan de Valdedo (el de Grandas de Salime, sin duda) y asimismo recuerdan la vieja indicación de L. Pérez Mínguez para las inmediaciones de Oviedo, que figura en Colmeiro (1885: 370). Especie, claro, con la que no es ordinario tropezar en la Cornisa Cantábrica. Véase lo dicho en AEDO \& al., 1985: 200. 


\section{Cytinus hypocistis (L.) L.}

Pr. Bojo (Allande), 29TPH79, leg. Aedo, 31-V-1987. Cita que parece ser la primera concreta para el occidente regional (cf. LAÍNZ, 1982: 31). Sobre Halimium alyssoides (Lam.) K. Koch, caso que también era el de la planta colectada por J. Delgado en Alles (cf. ARGÜELLES \& al., 1984: 7). A última hora, 13-V-1989, vuelve Aedo a dar con la especie pr. El Molino (Pesoz), dentro de la misma cuadrícula que más arriba consignamos.

\section{«Euphorbia palustris L. »}

Su cita de Moniello (Gozón), hecha en MAYOR \& al. (1974: 126) y reflejada en MAYOR \& DíAZ (1977: 250-252), tuvo por base FCO 2158, carente por de pronto de hojas; de semillas lisas, ciertamente, pero tan solo de c. $2 \mathrm{~mm}$... Bien claro está que la especie no alcanza estas regiones.

\section{«Aconitum anthora L. »}

Su presencia en la región ha sido ya descartada en alguna forma por «Flora iberica» (1: 234). Parece útil, no obstante, decir de modo expreso que no se tuvo por creíble cita como la hecha de Covadonga, por DíEz \& al. (1984: 191), al publicar un recuento cromosomático sin duda correspondiente al allí abundantísimo A. napellus L. subsp. vulgare Rouy \& Fouc.

\section{Ranunculus ololeucos Lloyd}

Especie cuya «bondad» propugnaremos hoy, tras haberla colectado hace seis lustros junto a Grandas de Salime, 29TPH78, 22-III-1959; y luego, cuidadosamente, no pocas veces más, en Galicia: pr. Puente-Olveira (Dumbría, La Coruña), 29TMH96, 6-V-1965; pr. Villar de Santos (Orense), 29TNG96, 28-III-1966; sobre La Cañiza (Pontevedra), 29TNG57, 30-III-1966; pr. Golada (Pontevedra), 29TNH83, 14-IV-1966; alto del Rodicio (sobre Maceda, Orense), 29TPG18, 25-IV-1966; Goyán (Tomiño, Pontevedra), 29TNG14, 21-V-1966. Velayos (1988: 117), desdiciéndose de lo aceptado en CiRUJANO \& al. (1987: 99-110), acaba de animarse a dar ese binomen por más o menos vergonzante sinónimo de $R$. peltatus Schrank subsp. fucoides (Freyn) Muñoz Garmendia, trinomen que finalmente adopta para lo que se había denominado $R$. peltatus subsp. saniculifolius (Viv.) C. D. K. Cook. No ha hecho recolección atlántica ninguna y nos parece muy posible que le haya desorientado Cook, al que ya se tildó en su día -cf. LAínZ, 1967: 3, nota 3-de conocer poco a fondo lo descrito por Lloyd. Es posible que tal especie no alcance la meseta, contra lo que «Flora iberica» supone. Tenemos también algunas dudas, vetustas -cf. 1. c.-, de que la uña de los «pétalos» realmente pueda ser amarilla en ololeucos auténtico; lo que, por de pronto había entrado en contradicción frontal, desde los tiempos de Freyn, con el epíteto restrictivo.

\section{R. trichophyllus Chaix}

Ante «Flora iberica» (1: 294-297), se ha hecho una cita picoeuropeana de la subsp. eradicatus (Laest.) C. D. K. Cook, tan solo referente al asturiano lago de las Moñetas, 30TUN58 (cf. FERNÁNDEZ BERNALDO DE QUIRÓS \& FERNÁNDEZ GARCía, 1987: 66). A continuación, hay quien ha decidido que todo lo picoeuropeano - al menos a partir de una prudente altitud- ha de adornarse con ese nombre (cf. PIZARRO, 1988: 49-51; RIVAS MARTíNEZ \& PIZARRO, 1988: 207; NAVA, 1988: 43-44). Este último autor atribuye a su planta pétalos de 5-7 mm... Véase, por otra parte, lo que acerca de la referida subespecie decía 
tiempo ha CooK (1966: 141), ante lo que toda la cautela en las determinaciones resulta
exigible.

\section{R. ophioglossifolius Vill.}

Dejemos aquí bien señalada su primera cita de Asturias (cf. LAÍNZ, 1989b: 585), que se adicionó a "Flora iberica» un tanto informalmente: pr. El Pandal (Villaviciosa), 30TUP02, Aedo, 20-V-1986.

\section{«Thalictrum tuberosum L. »}

Aunque «Flora iberica» (1: 391$)$ estuvo en este caso muy sobre aviso, el hecho de que GUINEA (1953: 400) mencione Avilés como localidad asturiana de la especie hace aconsejable una explicitación de que MA 149899 -Aterido leg., IX-1928- no corresponde a la misma. Caso diverso es el del pliego MA 41707, al que se refiere COLMEIRo (1885: 15) de modo muy explícito: sus dos etiquetillas dicen «Salcedo dedit anno 1807 y, al atribuir a la planta - bien determinada- una procedencia "ex Asturiae montibus», yerran como las otras parecidas a que venimos refiriéndonos -cf. LAÍNZ, 1987: 604.

Alyssum minutum Schlecht. ex DC.

Torrebarrio (San Emiliano, León), 30TTN56, Aedo, 10-V-1984; en calizas, a 1300 m. Del este de Orense, donde no es rara, se conoce ya desde los tiempos de MERINO (1905: 146). En LEB hay un pliego, correctamente determinado -n. ${ }^{\circ} 11645-$, de La Omañuela (Riello), 30 TTN54, al que se refiere M. A. Pérez García en su memoria doctoral inédita «Flora y vegetación de la comarca de Omaña» (Facultad de Farmacia, Santiago de Compostela).

Impreso el párrafo que antecede, recibimos un breve resumen de la memoria en cuestión -publicado en 1984-, cuyas págs. 9 y 15 se refieren a la especie que nos ocupa, sin concretar localidades pero dándola por muy difundida en esa zona leonesa y, en concreto, por novedad provincial.

\section{Reseda media Lag.}

Pr. Oviedo, 30TTP60 -como adventicia, posiblemente-, Aedo, 14-IV-1983. A ella corresponden los pliegos del cabo Peñas -FCO 1259-, 30TTP73, y del faro de San Juan de Nieva (Gozón), 30TTP63 -FCO 1260-, citados por MAYOR \& al. (1974: 31) como de $R$. phyteuma L. De Avilés hacia Galicia nuestro endemismo era bien conocido (cf. DUPONT \& DuPONT, 1956: 324). No hace mucho (cf. AEDo \& al., 1986: 60) que nos referimos a su avanzadilla distribucional en la costa montañesa.

\section{Cistus psilosepalus Sweet}

Chorín (Oviedo: parroquia de San Claudio), 30TTP60, brezales, a unos $170 \mathrm{~m}$. Aedo, 15-VI-1989. Localidad oriental extrema en Asturias, de momento al menos; la que de alguna forma enlaza con la de Grado, Peñaflor, en lo ecológico tal vez imprecisa (cf. GAY, 1836: 126-127).

\section{«Hypericum hyssopifolium Chaix»}

Cita de las más dificilmente creíbles -referente a Proaza- de las de G. MARTínEz (1976: 182); y eso, aunque MAYOR \& DiAZ (1977: 348) parezcan estribar en ella. En FCO, por fin, ha 
salido a flote su base material, que se intercala con el $\mathrm{n}^{\circ} 17128 \mathrm{y}$ que, desde luego, no corresponde a la especie. La vetusta indicación de Pérez Minguez recogida por COLMEIRO (1885: 536) tampoco es como para entusiasmar a nadie.

Sorbus mougeotii Soyer-Will. \& Godr.

Lo que así venimos llamando (cf. LAíNZ, 1976: 16; etc. ) está, decididamente, muy difundido en León y Asturias. Nuestras últimas recolecciones fueron las hechas en el puerto leonés de las Señales, a 1650 m, 29-VI-1983 -como en el caso que sigue, 30TUN17-, y en el coto asturiano de Reres, lugar que se llama Valdebezón - silíceo asimismo-, 18-VII-1987, a unos $1350 \mathrm{~m}$.

\section{Pyrus cordata Desv.}

Sierra del Castillo, pr. Pajares (Lena), 30TTN76, Aedo, 1-VIII-1987. Véase lo dicho por LAÍNZ (1979: 39); donde, por cierto, se hace una sola indicación provincial concreta (PiloñaPonga: 30TUN19). También es la especie difundida en Cantabria. Los frutos, contra lo que se ha dicho y repetido, no pueden llamarse nunca rojos.

\section{Prunus padus L.}

Puerto de San Isidro, en la zona denominada Entresierras (Aller), 30TUN07, Argüelles, J. González Alonso, González del Valle \& Laínz, 18-VI-1989, como a 1650 m. Se vio tan solo un arbusto, más bien raquítico, sobre una peña caliza. -Cf. ARGÜELLES \& al., 1984: 8.

\section{Coronilla glauca L. (=Jusl.)}

Hemos tenido curiosidad en ver el pliego asturiano a que se refirió GUINEA (1953:323). No es dudoso que la planta está bien determinada, excepcionalmente, por Cesáreo Martínez y que dio con la misma en Sotiello (Gijón), 30TTP72, el 3-VI-1915. Tampoco lo es que se trata de algo adventicio, traído por el ferrocarril carbonero: [junto al] disco [de la] vía, quiso poner la etiqueta.

\section{Vicia pubescens (DC.) Link}

No escasa en el concejo de Gijón: hacia Castiello (Bernueces), 30TTP82, Laínz, 4-VII-1985, y Santurio, ibidem, González del Valle \& Laínz, 6-VI-1986. Observada luego en Fenolleda (Candamo), 29TQJ31, Argüelles, González del Valle \& Laínz, 8-VI-1986. Aedo ahora impone tal nombre al pliego FCO 2026, de San Esteban de Morcín, 30TTN69, que figuraba como «tetrasperma» (cf. NAVARRO, 1977: 93); y da por admisible la determinación de otro, FCO 1998, más bien malo, procedente de una «escombrera en las inmediaciones de San Juan de Nieva (Gozón, Asturias), 8-VII-1973. Leg. et det. : Díaz y Navarro», al que no parece que podrá referirse la mención de MAYOR \& al. (1974: 125-126). Desestima, en cambio, determinaciones como la del pliego de Muriellos (Riosa), FCO 1999, al que se refirió NAVARRo (1977: 93) -se trata de V. hirsuta S. F. Gray-, sin haber dado con material ninguno de la Grandiella que se mencionaba simultáneamente. DíAZ (1977: 165) reitera la cita occidental y accidental de LAÍNZ (1968: 8), recordando las de los ovetenses y la tan conocida como vetusta de GAY (1836: 127).

De paso, digamos que Aedo asimismo rectifica la «Vicia tenuissima» del Aramo (cf. NAVARRO, 1977: 93), FCO 2020, que no es V. parviflora Cav. sino la común V. tetrasperma (L.) 
Schreber; y una «Vicia disperma DC.» de Trubia (FCO 16245, G. Martínez), que también ha resultado $V$. hirsuta, común a más no poder.

\section{Lathyrus bauhinii Genty}

Cf. LAİNZ (1987a: 542-543; 1989a: 557). Vemos ahora publicadas (cf. PUENTE, 1989: 236) citas occidentales de "Lathyrus filiformis (Lam.) Gay», altamente sospechosas. Localizado el pliego FCO 9407 -de Peña Blanca, 27-VII-1978, Fernández Prieto leg.-, queda clarísimo que la especie somedana es L. bauhinii Genty. Huelga ponderar el interés corológico de la constatación, a un lado el «político»-tal planta se hace también asturiana-, más el «depurativo» en lo que se refiere a filiformis.

«Malva alcea L. »

Cita de G. MARTÍnez (1976: 182; cf. MAYOR \& DíAZ, 1977: 452), quien acataba otra de Pérez Mínguez o Colmeiro (1885: 499) referente a las "cercanías de Oviedo», ciudad. Aunque no intercalados aún, vemos en FCO dos pliegos de Ventana, 12-VIII-1971 y 21-VII-1972, con la determinación susodicha. Corresponden a M. moschata, saltem s. 1., como es natural.

\section{Linum narbonense L.}

Picos Albos de Saliencia, bastante por debajo de las cumbres pero no poco por encima del Cerveriz, 29TQH36, Argüelles, González del Valle \& Laínz, 29-VI-1984, a unos 1900 m. Novedad provincial, según todas las apariencias. La cita de Pérez Mínguez o ColmeIro (1885: 487) de las "cercanías de Oviedo», así como la invocación (l. c.) de «Lag.»-muy explicable por sus actividades leonesas-, olvídense tranquilamente. La que se atribuye a Fernández Prieto sin concretar poco mi mucho (cf. IzCo \& al., 1985: 135), estamos en que sigue inédita.

«Geranium pratense L. »

Otra cita de Ventana que nos deslizaba en el catálogo provincial G. MARTiNEZ (1976: 180), con el agravante de haber añadido a la misma una de Leitariegos a todas luces errónea (cf. CARBÓ \& al., 1973: 276). MAYOR \& DIAZ (1977) tenían ya en este caso la prudencia de silenciar la especie. Los materiales de base, FCO 2066 y 15045, del 25-VI-1968 y 3-VI-1972, corresponden a $G$. sylvaticum, saltem s. 1.: estambres típicos, etc.

\section{Rhamnus cathartica L.}

Pr. Lavarejos (Ribera de Arriba), 30TTN69, Aedo \& P. García, 28-V-1987, matorral sobre calizas, a unos $300 \mathrm{~m}$. Debe recordarse la cita de VICIOSO (1946: 54), quien escribió, a secas: «Los Veyos (Asturias)»; 30TUN38, en toda hipótesis. Arbusto autóctono en la región, ciertamente; aunque raro, se diría.

Eryngium duriaei Gay ex Boiss.

Descrito de Leitariegos (Cangas del Narcea), fue ahora fotografiado por G. Moreno Moral -det. Aedo- en la braña de Llaneces (por encima de Caunedo, Somiedo), 29TQH27, 11-IX-1987, a unos $1400 \mathrm{~m}$. Localidad asturiana la más oriental, por el momento, de nuestro endemismo silicícola -cf. LAÍNZ, 1964: 196; 1969: 255; etc. 
Torilis elongata (Hoffmans. \& Link) Samp.

Santa Lucía (La Pola de Gordón, León), 30TTN85, González del Valle \& Laínz, 6-VII-1986. En sitio ruderal, sobre calizas, a $1050 \mathrm{~m}$. A la única localidad leonesa de que parece haberse citado (LAÍNZ, 1968: 17) corresponden las coordenadas 29TPH70. Véase también Rico, 1985: 414-415.

\section{Limonium humile Miller}

Vilavedelle (Seares, Castropol), 29TPJ51, Aedo, 20-X-1988. De la misma localidad citaba DíAZ (1976: 442) L. vulgare, por error cierto: FCO 14904 viene a confirmárnoslo, amén de que por allí no se ve otra cosa. Novedad provincial relevante (cf. LAínZ, 1976: 23).

Añadamos que, al revisar en 1987 nuestros materiales del grupo, el especialista M. Erben creyó ver $L$. x neumanii Salmon ( $=$ L. humile Miller x $L$. vulgare Miller) en alguna de nuestras muestras montañesas y galaicas, de localidades en que no conviven los progenitores. La cita de hoy viene a dar un poco de verosimilitud asimismo a esas determinaciones galaicas, por más que referentes a la playa de Baldayo (pr. Carballo, La Coruña), 29TNH29, y a la Puebla del Caramiñal, coruñesa igualmente, aunque todavía mucho más alejada: 29TNG01.

En cuanto a las citas de $L$. vulgare «subsp. serotinum (Reichenb. ) Gams» que vemos en ASEGINOLAZA \& al., 1985: 551, claro es que dependen aún de «Flora Europaea» (4: 42) o ERBEN (1978: 411); autor este último que rectificaba luego (1981: 504-505), aunque tal vez no plenamente, de manera bien explícita.

\section{Androsace halleri L.}

Tras una cuidadosa recolección de Argüelles, Delgado \& Laínz y, además, a la vista de una buena diapositiva que fue con los materiales, a tal especie lleva KRESS (1988) la colonia del Pico Huevo de Faro, 30TTN96 (Cármenes-Valdelugueros, León), sobre la que posteriormente, una vez más, López PACHECo (1988: 143-144) dice algo no acertado. Especie la nuestra que salta desde los Pirineos orientales a su notabilísima localidad cantábrica, la que por fin visitó Kress, en la buena compañía de Argüelles, el 14-VI-1988. A. laggeri Huet se acantona en el Pirineo central. A. cantabrica (Losa \& Montserrat) Kress -de los macizos de Peña Prieta y alto Campoo-sería, con toda verosimilitud, el alopoliploide.

\section{Pedicularis foliosa L.}

Majada de las Rubias, por encima del bosque de Valgrande (Lena), 30TTN76, Argüelles \& Laínz, 25-VII-1986, como a 1650 m. Nada general en estas regiones (cf. LAíNZ, 1962: 26; 1976: 27; AEDO \& al., 1986: 62); aunque hoy nuestra cita confirma, de alguna manera, la del Aramo que se basó en la recolección mierense del siglo XVIII. Una localidad asturiana ulterior, que abona el herbario Laínz, es la de Sotres (Cabrales), 30TUN58, donde abunda la especie como en algún punto del vecino término de Camaleño, fuera ya de la provincia: de ahí que la hipótesis de NAVA (1988: 116), en lo referente a Leresche \& Levier-Gandoger copia sin más, claro está-, no atine mucho.

\section{Bartsia spicata Ramond}

Observada por Aedo \& P. García pr. Toriello (Ribadesella), 30TUP31, en agosto de 1987, a $400 \mathrm{~m}$. Localidad occidental extrema, que sepamos. A la inicial de LAínZ (1959: 686) corresponden las coordenadas 30TUP50. 


\section{Scutellaria alpina L.}

Plenamente dentro de Asturias, abunda en los Picos Albos de Saliencia (Somiedo), 29TQH36, Argüelles, 29-VII-1973; y existe asimismo pliego colectado bajo el Portichín (Lena: Ubiña), 30TTN56, Lainz \& Lecubarri, 15-VIII-1981. Figuraban ya dos localidades tan asturianas como leonesas en LAÍNZ, 1961: 175 y 1963: 64.

\section{Stachys byzantina K. Koch}

Villamanín (León), 30TTN85, González del Valle, 25-VIII-1986. Ornamental alóctona que se naturaliza en ocasiones: vista de Cervera de Pisuerga (Palencia) en MA y MAF $-n^{\circ}$ 8610, exsiccata Auquier: leg. E. Fuertes \& Ladero, 21-VII-1976, «chênai claire»-; así como de Jaca (Huesca), «subespontánea» simplemente.

\section{Horminum pyrenaicum L.}

Frente a la majada de Mericueria, en el coto de Reres (Caso-Ponga), 30TUN17, donde se la vio, no poco difundida, sin colectarla: J. L. Aller, J. Delgado, Laínz \& J. Torío, 9-VIII-1986. Del no muy lejano puerto de San Isidro se conserva un pliego -iinédito?-, FCO 3361, F. Navarro, 7-VIII-1973. Localidades ésas intermedias entre las regionales, tan disyuntas, de los Picos de Europa, 30TUN58, y los lagos de Saliencia, 29TQH37. La ecología de la planta está en la base de los hechos.

Impreso el párrafo precedente, Argüelles añade que ya el 6-IX-1981 había observado él una muy nutrida colonia en la vertiente leonesa de San Isidro, junto al collado de Agujas, 30TUN07, como a $1700 \mathrm{~m}$. Novedad provincial, según todas las informaciones disponibles. A última hora, el 18-VI-1989, Argüelles, J. González Alonso, González del Valle \& Laínz lo ven difundidísimo en dicho puerto, dentro de Asturias (concejo de Aller), zona caliza denominada Entresierras, mucho más a mano; aunque otro pliego de allí visto en LEB -n. ${ }^{\circ} 8053-$, leg. E. Hernández, 15-VII-1977, precisa: «Puerto de San Isidro (León)». El Prof. Penas, por añadidura, nos dice que la especie alcanza lo alto del no lejano puerto leonés de las Señales, dentro de la misma cuadrícula que arriba se indica en el caso de Mericueria; y finalmente, que M. P. Fernández Areces lo citaba de la collada de Aralla, 30TTN65, en la memoria doctoral "Aportaciones al conocimiento de las comunidades vegetales de los paredones rocosos calizos de la Cordillera Cantábrica» - la que se imprimió defectuosamente y no quiso distribuir su autora.

\section{Viburnum opulus L.}

Aliseda cerca de Siejo (Alevia, Peñamellera Baja), 30TUN79, Aedo, V-1986. No colectado. Raro en la provincia. La cita de LAÍNZ (1979: 45) se refiere a localidad (30TTP70) muy distante de la de hoy.

Más común es -en zonas diversas- $V$. lantana L., aunque sus citas no sobreabunden (cf. LAínZ, 1958: 451). Se vio, por ejemplo, sobre Pendones (Caso) y en la parte cabraliega de los Picos -donde, hacia el Collado Vallejo, sube a $1500 \mathrm{~m}-$. Observado recientemente (21-IX-1987), por Aedo, en las brañas de Mumián (coto de Buenamadre, Somiedo), 29TQH27, a unos 1600 m. En Peña Redonda (Palencia), digámoslo de pasada, Laínz lo tiene visto a $1750 \mathrm{~m}$.

\section{Lonicera caprifolium L.}

Vegadeo, 29TPJ51, roquedo silíceo - un solo ejemplar-, Aedo, 8-IV-1989. Planta escapada, evidentemente, de cultivo jardineril. 


\section{Campanula scheuchzeri Vill. (?)}

Por encima del puerto de Tarna, 30TUN17, así en León como en Asturias, no escasa en brezales y pastos ácidos, a unos 1700 m. Legerunt C. Almendral, Argüelles, González del Valle \& Laínz, 30-VIII-1987.

Novedad asturiana, en toda hipótesis y según toda evidencia: las citas de Arrieu y Lascombes a que se refiere NAVA (1988: 123) eran respectivamente montañesa e inconcreta, hechas ambas en listas de cariz a que nos hemos referido en ocasiones múltiples.

Como de costumbre, nuestra recolección fue cuidadosa: rizomas inclusive. La planta no difiere de la de San Glorio, tetraploide, que dirigimos a Küpfer (cf. LAÍNZ, 1976: 34). Los capullos eran, por supuesto, cabizbajos. Las corolas, no tan grandes como las de la planta campurriana en la que nos hizo Küpfer sus recuentos hiperhexaploide y hexaploide (cf. LAÍNZ, 1979: 45).

Acabamos de ver una cita bejarana (SÁNCHEZ \& al., 1987: 104) que resulta no poco sorprendente. Ciertamente, Llamas (1984: 122) las hizo leonesas, del Teleno; donde NIETO FELINER (1985) no ha visto cosa ninguna del grupo.

«Senecio nemorensis L. subsp. bayonnensis Boiss. »

La cita de PEINADO \& MARTÍNEZ PARRAS (1982: 534), hecha con alguna conciencia de su poca verosimilitud, es del todo inverosímil.

\section{Centaurea lagascana Graells}

Por lo que a la provincia de Asturias hace, casi todo lo que vemos en la distribución de FERNÁNDEZ CASAS \& SUSANNA (1986: 97) parece sujeto a objeciones. Ese maltratado "Alto de los Pinos» que dicen Font Quer \& Rothmaler -visitantes de un solo día-, si "fide Laínz» corresponde a León, ¿por qué no pasó al epígrafe correspondiente? Lo de «Campomanes (Lena), Merxmüller \& Lippert, VII-1975 (M)» será textual, pero increíble: planta colectada muy por encima y muy lejos de Campomanes, muy probablemente leonesa... Lo de «Torrebarrio - Tuiza (Lena)» también huele a flagrante inexactitud -en este caso, de celtíberos, no de tudescos-, puesto que Torrebarrio corresponde a San Emiliano (León) y dista no poco de las Tuizas de Arriba y de Abajo, ya en la vertiente que ahora nos ocupa, la de Asturias. Afortunadamente, los autores de la monografia echan por delante (1. c.: 96) que la planta es "propia de la solana de la Cordillera Cantábrica», lo que les ha dejado a salvo de toda posible crítica de mayor entidad. Su mapa estará hecho-suponemos- a ojo de buen cubero, en lo que a estas localidades concierne.

\section{Hypochoeris maculata L.}

Sobre Tonín (Villamanín, León), 30TTN86, ladera silícea, como a $1500 \mathrm{~m}$. Legerunt Argüelles, González del Valle \& Laínz, 2-VIII-1987.

Escasean mucho las citas del noroeste; aunque, de León, topamos con la de CARBÓ \& al. (1973: 282, «Villargusán», término municipal de San Emiliano, "en pastizales de siega»), más las de Romero (1983: 121, "Miñera de Luna», término municipal de los Barrios de Luna; indicaciones ecológicas muy otras), LóPEZ PACHECO (1988: 186) y PÉREZ MORALES (1988: 169-170).

\section{Leontodon autumnalis L.}

Pr. Castropol, 29TPJ51, en herbazales subsalsuginosos, Laínz, 6-VII-1973. Planta normalmente desarrollada, como la que tenemos de La Espiñeira (Foz, Lugo), 29TPJ42; no «sehr 
schmalblättrige, meist einköpfige Form», como la que se habría visto en las junqueras de Ribadeo (cf. LƯDI, 1956: 298). A pesar de su hábitat, no muy normal acaso, estamos en que se trata de la especie linneana, típica. Bien posible novedad provincial, dada la confusión que viene reinando en el grupo. Cf., v. gr., lo dicho por LAÍNZ (1976: 37) a propósito de $L$. carpetanus Lange.

\section{Taraxacum L.}

Hace algunos años que dirigimos diversos materiales genéricos, para su determinación, al hoy difunto C. I. Sahlin ( $†$ 1987). A raíz casi de su fallecimiento, el Botanical Museum de Göteborg -con las más correctas excusas- nos devolvió esos materiales, algunos un tanto estropeados; otros, a medio estudiar. Publiquemos las determinaciones firmes y las aproximativas del especialista en cuestión - «veris.»= verisimiliter-, dejando a un lado las que suponen ciertamente nom. nud., o sea «sp. nov.» indescrita. Son, geográficamente ordenadas: T. maculosum Richards, "veris.», Quintanilla de Rucandio (Valderredible, Cantabria), 30TVN35, in graminosis plus minusve calcareis, ad $700 \mathrm{~m}, 28-\mathrm{V}-1983 ; T$. vetteri v. Soest, Horcada de Don Carlos (Picos de Europa, in parte centrali, ditione ovetensi), 30TUN58, alt. $2411 \mathrm{~m}, 20-\mathrm{VIII}-1962 ;$ T. hydrophilum v. Soest, "veris. ", in silva dicta Peloño (Ponga), 30TUN27, ad $1100 \mathrm{~m}, 20-\mathrm{IV}-1959 ;$ T. braun-blanquetii v. Soest, in summa Peña Ubiña (León-Asturias), 30TTN76, ad $2400 \mathrm{~m}, 23-\mathrm{VI}-1959$, atque in rupibus item calcareis montium dictorum Aquilianos (León), 29TPH90, ad $1500 \mathrm{~m}, 17-\mathrm{VI}-1972 ; T$. hyoseridifolium Arv.-Touv. \& Marc. d'Aym., Picos Albos de Saliencia (Somiedo), 29TQH36, in rupestribus calcareis ad 1650 et 1700 m, 28-VI-1961, atque "veris.» loco dicto Gargantada del Hoyo Grande (Picos de Europa, in parte centrali, ditione legionensi vel santanderiensi), 30TUN48, ad 2300 m, 1-IX-1977; T. bargusicum Sahlin, supra Caboalles de Arriba (Villablino, León), ad 1300 m, 16-VI-1972; T. badiophyllum Sahlin, Sierra de Ancares (in ditione lucensi), 29TPH74, ad $1450 \mathrm{~m}, 22-\mathrm{V}-1965$.

\section{Reichardia picroides (L.) Roth}

Onón (Tornón, Villaviciosa), 30TUP02, cantera en total abandono, Aedo, 1-VI-1986. Una referencia incidental (cf. LAÎNZ, 1973: 196) hubo de ser confirmada en MA recientemente, ante las dudas que Nava nos insinuó. Luego, resulta que también él ha visto esa planta en la Campa de Torres - pr. Gijón-, evidentemente aquí alóctona. Tal es el caso en el barrio de San Cristóbal, de La Coruña, donde nos la colectó el P. Gómez Vigide; pero no en la mayoría de sus localidades montañesas (cf. DUPONT, 1955: 439), aunque tal vez sí en otras.

\section{Pilosella galliciana (Pau) Laínz}

Pico Huevo de Faro (Cármenes-Valdelugueros, León), 30TTN96, no abundante, sobre pizarras, como a 2100 m. Legerunt Argüelles, J. Delgado \& Laínz, 5-VIII-1984, det. B. de Retz. Cita la más occidental dentro de León. Recordemos, ya dentro de Cantabria, la de Peña Prieta, 30TUN56, que figura en LAÍNZ \& LORIENTE, 1983: 413. Bajo el nombre - defendiblede P. lactucella subsp. bergidensis (Laínz) Laínz, ha sido luego señalada en Braña Caballo por PUENTE \& al., 1985: 298.

\section{Hieracium saliencianum de Retz, sp. nov.}

«Phyllopodum, gymnopodum. Caulis $15-25 \mathrm{~cm}$, glaberrimus (superne subfloccosus), 1 vel 2 cephalus. Folia margine et costa dorsali distincte glandulosa, glandulis brevibus et tenellis, pilis et floccis nullis. Folia radicalia 4-5, prasino-glauca, supra submaculata, exteriora late ovata et 
parce dentata, interiora elliptica vel oblongo-lanceolata, \pm grosse dentata vel inferne subpinnato-dentata. Folium caulinum 0 vel 1 reductum. Pedicelli \pm floccosi, sparsim glandulosi, epilosi. Involucrum 10-13 mm; squamis acutiusculis, viridi-atris, parce floccosis, modice glandulosis, epilosis. Alveoli receptaculi dentibus glabris. Ligulae luteae, apice glabrae. Styli lutei. Achaenia 3,5-4 mm, brunneo-nigra.

Espagne: Picos Albos de Saliencia (Somiedo, prov. Asturias), 29TQH36, rochers calcaires, alt. 1950 m. Leg. J. M. Argüelles, J. M. González del Valle \& M. Laínz, 29 juillet 1984.

Holotypus in herb. B. de Retz ( $\left.\mathrm{n}^{\circ} 86467\right)$, isotypi in herb. M. Laínz.

Le port de la plante rapelle celui d'un petit Hieracium pallidum Biv.-Bern. ou $H$. praecox Sch.-Bip., mais en diffère très essentiellement par l'absence à peu près complète de tout poil sur l'ensemble de la plante, et la présence de fines glandes sur le bord et la nervure dorsale des feuilles, ainsi que sur les écailles involucrales. Cette particularité de l'indument avait déjà été constatée (1905) sur une plante des rochers calcaires des Alpes du Dauphiné et à la même altitude de $1950 \mathrm{~m}$ : Hieracium chaixianum Arvet-Touvet et Gautier, distribuée par eux sous le $n^{\circ} 870$ de leur Hieraciotheca Gallica. Toutefois la plante des Picos Albos de Saliencia diffère de celle des Alpes du Dauphiné, par plusieurs caractères, tels que la forme et la couleur des feuilles, l'inflorescence moins développée, etc. On peut-se demander si $H$. saliencianum ne serait pas un vicariant cantabrique du $H$. chaixianum du Dauphiné?» (B. de Retz in litteris).

Planta que parece muy localizada pero no escasa en la zona donde se la vio de manera repetida. En el herbario Laínz hay muestras colectadas ya el 8-VII-1963 y 29-VII-1975. Tanto Argüelles como González del Valle conservan algún material de la recolección que menciona el protólogo, aunque no visto por de Retz. Ambos colectaron el 3-VIII-1985 cipselas que fueron puestas inútilmente a germinar en el Real Jardín Botánico de Madrid.

\section{H. merxmuelleri de Retz}

Pr. Villanueva (Santo Adriano), en calizas cortadas a pico, 30TTN59, a unos $200 \mathrm{~m}$. De Retz, frente a una muestra difícilmente determinable, colectada por Laínz el 1-VIII-1969, y ante altitud que le sorprendía - la de su localidad clásica es de $1450 \mathrm{~m}-$, exigió reherborización; la que finalmente, no sin dificultades, pudo llevar a cabo Argüelles el 1-VII-1984, en perfectas condiciones. La pedinosis, aquí, es fenómeno habitual, que desorienta de vez en cuando a los extranjeros.

Acabamos de señalar esa planta en Galicia (cf. LAÍNZ, 1989b: 586).

\section{H. lainzii de Retz}

Peña Ubiña la Pequeña -dentro, pues, de León-, 30TTN56, Argüelles \& Laínz, 10-VIII-1983, como a $2000 \mathrm{~m}$. Bernard de Retz det.: «Plante plus grêle que dans le type de Tolibia de Abajo [Valdelugueros: 30TUN05] (moindre développement des feuilles caulinaires), probablement du fait de l'altitude de la récolte. Parfaitement conforme pour le reste (notamment réceptacle à peu près dépourvu de cils!)».

Frente a la tesis picoeuropeana de NAVA (1988: 136-137), decidimos publicar hoy algunas otras determinaciones de nuestro corresponsal de Retz que se refieren a los altos niveles del triple macizo, asturianas, cántabras o leonesas: $H$. cerinthoides L., collado de la Padiorna, 9-VIII-1962; H. mixtiforme Arv.-Touv. subsp. ellipsocerinthe (Arv.-Touv. ) Zahn, Canal de Remoña, 31-VII-1983; H. mixtum Froelich subsp. mixtum, Horcados Rojos, 13-VIII-1961 -el monógrafo ZAHN (1921: 170) lo citó de Ándara-, y subsp. bombycinum (Boiss. \& Reuter) Zahn, sobre la Canal de la Henduda, 9-VII-1982, más Collada de Remoña, 31-VII-1983; correspondiendo también a la última subespecie, "très probablement», una recolección de Argüelles que se hizo poco bajo la cumbre de la Torre de Cerredo, el 20-IX-1970. De 
Pilosella officinarum F. W. Schultz \& Schultz Bip. -syn. Hieracium pilosella L.-, se citó en ARGÜELLES \& al. (1984: 14) una subespecie picoeuropeana y asturiana, de altitud-Amuesa-, que parece habérsele pasado a Nava, quien por otra parte hiló poco fino en la grey. Otras recolecciones de Laínz, que difícilmente podrán ser llevadas a tal especie por muy «sensu lato» que se la conciba, fueron hechas, en los Picos, por debajo de los $1500 \mathrm{~m}$.

\section{Alisma lanceolatum With.}

Pr. Panes, (Peñamellera Baja) 30TUN79, Aedo, 3-VII-1987; pr. Lugones (Siero), 30TTP71, Aedo, 24-VI-1989. No parece haberse citado aún de la provincia. Sí hay citas montañesas (cf. LAÍNZ, 1973: 196-197; LAÍNZ \& LoRIENTE, 1983: 413; AEDO \& al., 1984: 137).

\section{Allium oleraceum L.}

Pombayón (Beyos, Ponga), 30TUN38, Laínz, 20-VIII-1969, y El Pandal (Villaviciosa), 30TUP02, Aedo, VIII-1986. Tan solo parecen preexistir las citas provinciales de PAU (1893: 76), 30TTN76, LAÍNZ (1959: 689), 29TQH26, y NAVARRO (1982: 46), 30TTN69.

\section{A. scorzonerifolium Desf.}

Monasterio de Coto (Cangas del Narcea), 29TPH87, Aedo, 11-VI-1986. No vemos indicación provincial ninguna.

\section{Carex disticha Hudson}

Puerto de Somiedo, 29TQH27, Aedo, M. Luceño \& P. Vargas, 13-VI-1988. Novedad provincial. De León, sí tenemos a la vista citas, más o menos incidentales, atinadas acaso. Cf. AEDo \& al. (en prensa).

\section{Poa infirma Kunth}

Pr. Bojo (Allande), 29TPH79, Aedo, 5-IV-1987. Segunda cita provincial, siendo la precedente (cf. HERRERA \& al., 1988: 327), un tanto inconspicua, del concejo costero de Soto del Barco. Publicamos ahora citas montañesas (cf. AEDO \& al., en prensa). Véase también lo que había dicho HERNÁNDEZ CARDONA, 1976: 32 y 1978: 50-59, quien recoge las orensanas de LAínZ, 1967: 49.

\section{Cephalanthera rubra (L.) L. C. Rich.}

Pr. Agüera (Belmonte), 29TQH28, Argüelles \& E. Reigada, 10- V-1987. Bosque sobre calizas, a unos $500 \mathrm{~m}$. Novedad provincial. Tras la cita lebaniega de Pereda (in LAínZ, 1970: 44) que se les pasó a PINTO DA SilVA \& TELES (1971:5) se han publicado para estas regiones la del Campoo montañés que se debe a Herrá (cf. AEDo \& al., 1984: 138), una segunda lebaniega (cf. RIVAS MARTÍNEZ \& al., 1984: 81) y las burgalesas de LAÍNZ (1974b: 20) y GALÁN (1986: 67), así como, al menos, una leonesa, de PÉREZ MorAles (1988: 205) -hay en LEB ulterior material colectado en la provincia y era de zona limítrofe la protoindicación orensana (LAÍNZ, 1967: 45).

P.S. En prensa ya el trabajo, hemos tenido la posibilidad -que agradecemos cordialmente al Prof. Penas y colaboradores- de hacer algunos retoques al mismo en el herbario LEB. Sobre Dianthus queda claro, en primer término, que son referibles a brachyanthus los de Santa Lucía (LEB 11256 y 29984, 12-VII-1977) determinados como hispanicus; pero no el de Quintanilla de Flórez (LEB 19465, 19-VI-1983), que resulta, iluminadoramente, un típico D. laricifolius 
Boiss. \& Reuter, el primero visto por nosotros de la provincia leonesa. También parece corresponder a la referida subsp. laricifolius un pliego que arreo nos mostró el Prof. Penas (LEB 17949), colectado por él mismo en su natal Valencia de Don Juan el 15-VII-1977. Volveremos en oportunidad próxima sobre tales asuntos.

A ultimísima hora damos con Thelypteris palustris Schott en los llamados lagos de Silva (Tapia de Casariego), 29TPJ62, Aedo \& J. Muñoz, 29-VII-1989, localidad medianera entre las montañesas y lucenses. Lo que a nuestro juicio, no apuntala mucho ni poco informaciones como la que atribuye a Lázaro el tan crédulo Colmeiro.

\section{Bibliografía}

Aedo, C., C. Herrá, M. Lainz, E. Loriente \& J. Patallo (1984). Contribuciones al conocimiento de la flora montañesa, III. Anales Jard. Bot. Madrid 41(1): 125-141.

Aedo, C., C. Herrá, M. Lainz, E. Loriente, G. Moreno Moral \& J. Patallo (1985). Contribuciones al conocimiento de la flora montañesa, IV. Anales Jard. Bot. Madrid 42(1): 197-213.

Aedo, C., C. Herrá, M. Lainz, E. Loriente, G. Moreno Moral \& J. Patallo (1986). Contribuciones al conocimiento de la flora montañesa, V. Anales Jard. Bot. Madrid 43(1): 57-64.

Aedo, C., C. Herra, M. Laínz \& G. Moreno Moral. Contribuciones al conocimiento de la flora montañesa, VII. Anales Jard. Bot. Madrid 47(1), en prensa.

Argüelles, J. M., J. Delgado \& M. Lainz (1984). Contribuciones al conocimiento de la flora de Asturias. Bol. Inst. Estud. Asturianos, ser. C., 33: 3-14.

aseginolaza, C., D. Gómez, X. Lizaur, G. Montserrat, G. Morante, M. R. Salaverria, P. M. Uribe-Echebarrila \& J. A. Alejandre (1985). Catálogo florístico de Álava, Vizcaya y Guipúzcoa. Vitoria.

Báscones, J. C., A. Ederra, A. Pérez Losantos \& L. M. Medrano (1982) Pteridófitos de Navarra. Collect. Bot. (Barcelona) 13(1): 19-35.

CARBó, R., M. MAYOR, J. ANDRÉS \& J. M. Losa (1973). Aportaciones al catálogo florístico de la provincia de León. An. Fac. Veterin. (León) 18: 224-352.

Catalán, P. \& I. Aizpuru (1989). Atlas de los pteridófitos de Navarra. Munibe 40: 99-116.

Cirujano, S., P. Pascual \& M. Velayos (1987). Aportación al conocimiento de Ranunculus peltatus Schrank subsp. saniculifolius (Viv.) C. D. K. Cook, y su comportamiento fitosociológico. Trab. Dep. Bot. (Madrid) 13: 99-110.

Cook, C. D. K. (1966). A monographic study of Ranunculus subgenus Batrachium (DC.) A. Gray. Mitt. Bot. München 6: 47-273.

DiAZ, T. E. (1976). La vegetación del litoral occidental asturiano. Rev. Fac. Ciencias (Oviedo), n. s., 16: $369-545$.

DiAZ, T. E. (1977). Sobre la flora vascular del litoral occidental asturiano. I (de Equisetaceae a Euphorbiaceae). Bol. Inst. Estud. Asturianos, ser. C., 22: 109-185.

DiAZ, T. E. \& E. PuEnTE (1985). Avance sobre la pteridoflora leonesa. An. Biol. (Murcia) 1: 267-299.

Diez, M. J., J. PASTOR \& I. FernÁndEZ (1984). Números cromosomáticos de plantas occidentales. Anales Jard. Bot. Madrid 41(1): 191-194.

Dupont, P. (1955). Contribution à la flore du Nord-Ouest de l'Espagne (II). Bull. Soc. Hist. Nat. Toulouse 90: 429-440.

Dupont, P. \& S. DuPONT (1956). Additions à la flore du Nord-Ouest de l'Espagne (I). Bull. Soc. Hist. Nat. Toulouse 91: 313-334.

Erben, M. (1978). Die Gattung Limonium im südwestmediterranen Raum. Mitt. Bot. München 14: 361-631.

ERbEN, M. (1981). Bemerkungen zur Taxonomie der Gattung Limonium II. Mitt. Bot. München 17: 485-510.

Fernández Bernaldo de Quirós, C. \& E. Garcia Fernández (1987). Lagos y lagunas de Asturias. Salinas (Castrillón, Asturias).

FERnÁndez CASAS, J. \& A. SusAnna (1986). Monografia de la sección Chamaecyanus Willk. del género Centaurea L. Treballs Inst. Bot. Barcelona 10: 1-174.

Fernández Prieto, J. A., T. E. Diaz\& J. M. Carballo (1982). Anotaciones sobre la flora astur. Bol. Inst. Estud. Asturianos, ser. C., 30: 23-40. 
GaLÁN, P. (1986). Datos previos para un catálogo de las orquídeas burgalesas. Anales Jard. Bot. Madrid 43(1): 65-82.

García GonZÁleZ, M. A. (1988). Efectos de las repoblaciones con pinos en la clímax de la Quercetea ilicis mediterránea leonesa. León.

GAY, J. (1836). Duriaei iter asturicum botanicum, anno 1835 susceptum. Ann. Sc. Nat., Bot., sér. 2, 6: 113-137, 213-225, 340-355.

GuineA, E. (1953). Geografia botánica de Santander. Santander.

Herrera, M., C. Aedo, T. E. Díaz \& J. A. Fernández Prieto (1988). Una nueva asociación cantábrica de la clase Polygono-Poetea annuae: Poo annuae-Spergularietum salinae. Acta Bot. Malacitana 13: 326-332.

Herrero, L., M. E. Garcia González, E. Puente \& A. Penas (1988). Aportaciones palentinas al Atlas Pteridológico de la Flora Ibérica y Balear 10 x 10. Acta Bot. Malacitana 13: 365-370.

Hernández Cardona, A. M. (1978). Estudio monográfico de los géneros Poa y Bellardiochloa en la Península Ibérica e Islas Baleares. Diss. Bot. 46: 1-365.

Izco, J., J. Guitián, J. Amigo \& J. Rodriguez Oubiña (1985). Apuntes sobre la flora gallega, 2. Trab. Compostel. Biol. 11: 131-140.

Kress, A. (1988). Primulaceen-Studien 9. Androsace halleri L. in der westlichen Cordillera Cantábrica. Gröbenzell bei München.

LAínZ, M. (1958). Aportaciones al conocimiento de la flora cántabro-astur, II. Collect. Bot. (Barcelona) 5(2): 429-460.

LAinZ, M. (1959). Aportaciones al conocimiento de la flora cántabro-astur, III. Collect. Bot. (Barcelona) 5(3): 671-696.

LAinz, M. (1961). Aportaciones al conocimiento de la flora cántabro-astur, V. Bol. Inst. Estud. Asturianos, ser. C., 3: 147-186.

LaínZ, M. (1962). Aportaciones al conocimiento de la flora cántabro-astur, VI. Bol. Inst. Estud. Asturianos, ser. C., 5: 3-43.

LAinZ, M. (1962). Aportaciones al conocimiento de la flora cántabro-astur, VI. Bol. Inst. Estud. Asturianos, ser. C., 5: 3-43.

LAínZ, M. (1963). Aportaciones al conocimiento de la flora cántabro-astur, VII. Bol. Inst. Estud. Asturianos, ser. C., 7: 35-81.

LAínZ, M. (1964). Aportaciones al conocimiento de la flora cántabro-astur, VIII. Bol. Inst. Estud. Asturianos, ser. C., 10: 173-218.

LAínZ, M. (1967). Aportaciones al conocimiento de la flora gallega, V. Anales Inst. Forest. Invest. Exp. 12: $1-51$.

LAinZ, M. (1968). Aportaciones al conocimiento de la flora gallega, VI. Madrid.

LAínz, M. (1969). In Floram Europaeam animadversiones. Candollea 24: 253-262.

LAínZ, M. (1970). Aportaciones al conocimiento de la flora cántabro-astur, IX. Bol. Inst. Estud. Asturianos, ser. C., 15: 3-45.

LaínZ, M. (1973). Aportaciones al conocimiento de la flora cántabro-astur, X. Bol. Inst. Estud. Asturianos, ser. C., 16: 159-206.

Lainz, M. (1974a). De Petrocoptidibus cantabricis ulteriores notulae. Collect. Bot. (Barcelona) 9: 191-194.

LAinZ, M. (1974b). Aportaciones al conocimiento de la flora gallega, VIII. Madrid.

Lainz, M. (1976). Aportaciones al conocimiento de la flora cántabro-astur, XI. Bol. Inst. Estud. Asturianos, ser. C., 22: 3-44.

LAínZ, M. (1979). Aportaciones al conocimiento de la flora cántabro-astur, XII. Bol. Soc. Brot., sér. 2, 53: 29-54.

Laínz, M. (1982). Mis contribuciones al conocimiento de la flora de Asturias. Oviedo.

LaínZ, M. (1987a). Lathyrus bauhinii Genty en la Cordillera Cantábrica. Anales Jard. Bot. Madrid 44(2): 542-543.

Lainz, M. (1987b). De re chorologica, nova et vetera. III. Anales Jard. Bot. Madrid 44(2): 603-604.

LainZ, M. (1989a). Algo más, a propósito de Lathyrus bauhinii Genty. Anales Jard. Bot. Madrid 45(2): 557.

Lainz, M. (1989b). De re chorologica, nova et vetera. IV. Anales Jard. Bot. Madrid 45(2): 585-586.

LAinZ, M. \& E. LoRIENTE (1982). Contribuciones al conocimiento de la flora montañesa. Anales Jard. Bot. Madrid 38(2): 469-475.

LASTRA, J. J. \& M. MAYOR (1980). Nota florística sobre Grado y sus contornos. Rev. Fac. Ciencias (Oviedo), n. s., 17-19: 309-315. 
LóPEZ GonZÁlEZ, G. (1987). Notas referentes al género Rumex. Anales Jard. Bot. Madrid 44(2): 580-591.

LóPEZ GonZÁlez, G. (1988). El nombre correcto de Rumex alpinus auct. et L. (1759), non L. (1753) (Polygonaceae). Anales Jard. Bot. Madrid 45(1): 368-369.

LÓPEZ PACHECO, M. J. (1988). Flora y vegetación de las cuencas alta y media del río Curueño (León). León.

LÜDI, W. (1956). Floristische Neufunde von Blütenpflanzen, gemacht auf der I. P. E. durch Spanien 1953. Veröffentl. Geobot. Inst. Rübel (Zürich) 31: 287-298.

Llamas, F. (1984). Flora y vegetación de la Maragatería (León). León.

Llamas, F., R. CARBó \& J. ANDRÉs (1985). Contribución al conocimiento de la flora de León (España). Lazaroa 6: 283-285.

Martínez, G. (1976). Observaciones ecológicas y florísticas sobre el Puerto de Ventana. Rev. Fac. Ciencias (Oviedo), n. s., 16: 145-204.

MAYOR, M. \& T. E. DíAZ (1977). La flora asturiana. Salinas (Castrillón, Asturias).

MAYOR, M., T. E. Diaz \& F. NAVARro (1974). Aportación al conocimiento de la flora y vegetación del Cabo de Peñas (Asturias). Bol. Inst. Estud. Asturianos, ser. C., 19: 93-154.

Mayor, M., H. Nava, J. R. Alonso Fernández \& M. A. Fernández Casado (1982). Notas florísticas y ecológicas sobre la flora ibérica (III). Rev. Fac. Ciencias (Oviedo), n. s., 22: 11-20.

Merino, B. (1906). Flora descriptiva é ilustrada de Galicia, vol. 2. Santiago de Compostela.

Montserrat, P. (1988). Novitates in genere Petrocoptis A. Braun. Anales Jard. Bot. Madrid 45(1): 362.

NAVA, H. (1988). Flora y vegetación orófila de los Picos de Europa. Ruizia 6: 1-243.

Nava, H. \& M. L. Vera DE la Puente (1987). Acerca de la presencia de Rumex alpinus L. en la Cordillera Cantábrica. Anales Jard. Bot. Madrid 44(2): 536-537.

NAVARro, F. (1977). Datos para el catálogo florístico del Aramo y sus estribaciones (Asturias). I. De Equisetaceae a Linaceae. Bol. Inst. Estud. Asturianos, ser. C., 22: 45-108.

Navarro, F. (1982). Datos para el catálogo florístico del Aramo y sus estribaciones (Asturias). V: monocotiledóneas, excepto poáceas y ciperáceas. Stud. Bot. (Salamanca) 1: 42-58.

Nieto Feliner, G. (1985). Estudio crítico de la flora orófila del suroeste de León: Montes Aquilianos, Sierra del Teleno y Sierra de la Cabrera. Ruizia 2: 1-239.

PAU, C. (1893). Plantas españolas recogidas el año pasado por mi distinguido amigo y colega Sr. A. E. Lomax, de Liverpool, según muestras enviadas por el mismo. Actas Soc. Esp. Hist. Nat. 1893: 77-89.

Peinado, M. \& J. M. Martinez Parras (1982). Notas corológicas sobre las provincias Orocantábrica y Atlántica. Anales Jard. Bot. Madrid 38(2): 532-534.

PÉrez Morales, C. (1988). Flora y vegetación de la cuenca alta del río Bernesga. León.

Pérez García, M. A. (1984). Flora y vegetación de la comarca de Omaña (León). Resumen de tesis doctoral. Facultad de Farmacia. Santiago de Compostela.

Pinto Da Silva, A. R. \& A. N. Teles (1971), in A. R. Pinto Da Silva (ed.), Treze espécies e subespécies novas para a flora de Portugal. Agron. Lusit. 33: 1-24.

PizarRo, J. (1988). Ranunculus trichophyllus Chaix subsp. eradicatus (Laest.) C. D. K. Cook en los Picos de Europa (Cantabria, España). An. Biol. (Murcia) 13: 49-51.

Preston, C. D. \& P. D. Sell (1989). The Aizoaceae naturalized in the British Isles. Watsonia 17(3): 217-245.

Puente, E. (1989). De plantis legionensibus. Notula XIII. Stud. Bot. (Salamanca) 7: 235-241.

Puente, E., C. Pérez Morales \& M. J. Lopez Pacheco (1985). Nuevos datos para la flora leonesa. Lazaroa 6: 297-299.

Puente, E., C. Pérez Morales, M. J. López Pacheco \& A. Penas (1988). Aportaciones leonesas al Atlas Pteridológico Ibérico y Balear 10 x 10. An. Biol. (Murcia) 13: 370-373.

Rico, E. (1985). Aportaciones y comentarios sobre la flora del centro-oeste español. Anales Jard. Bot. Madrid 41(2): 407-423.

Rivas Martínez, S., T. E. Diaz, J. A. Fernández Prieto, J. Loidi \& A. Penas (1984). La vegetación de la alta montaña cantábrica - Los Picos de Europa. León.

Rivas Martínez, S. \& J. Pizarro (1988). Datos sobre la vegetación y biogeografia de los Picos de Europa. Acta Bot. Malacitana 13: 201-208.

Romero Rodriguez, C. M. (1983). Flora y vegetación de la cuenca alta del río Luna (León). Monografias ICONA 29: 1-273.

Salvo, A. E., B. Cabezudo, L. España, T. E. Diaz, J. Iranzo, J. Izco \& C. Prada (1984). Atlas de la pteridoflora ibérica y balear. Acta Bot. Malacitana 9: 105-128. 
SÁncheZ, J., F. Amich \& F. Herrero (1987). Aportaciones corológicas a la flora centro-occidental. Bol. Soc. Brot., sér. 2, 59: 97-112.

Velayos, M. (1988). Acotaciones a Ranunculus subgénero Batrachium (DC.) A. Gray: Tratamiento taxonómico general y estudio de la variabilidad de $R$. peltatus. Anales Jard. Bot. Madrid 45(1): 103-119.

Vera de la Puente, M. L. (1985). Evolución de los brezales quemados en la Cordillera Cantábrica. Bol. Inst. Estud. Asturianos, ser. C., 34: 69-77.

Vicıoso, C. (1946). Notas sobre la flora española. Anales Jard. Bot. Madrid 6(2): 5-92.

ZAHN, K. H. (1921), in H. G. A. ENGLER, Pflanzenreich (Heft 75). IV. 280. Compositae-Hieracium. Sect. I. Glauca - Sect. VII Vulgata (Anfang). Leipzig. 